\title{
Reduced blood brain barrier breakdown in P-selectin deficient mice following transient ischemic stroke: a future therapeutic target for treatment of stroke
}

Albert Y Jin', Ursula I Tuor², David Rushforth², Jaspreet Kaur ${ }^{1}$, Robert N Muller ${ }^{3}$, Jodie Lee Petterson', Sébastien Boutry ${ }^{3}$, Philip A Barber ${ }^{1 *}$

\begin{abstract}
Background: The link between early blood- brain barrier (BBB) breakdown and endothelial cell activation in acute stroke remain poorly defined. We hypothesized that P-selectin, a mediator of the early phase of leukocyte recruitment in acute ischemia is also a major contributor to early BBB dysfunction following stroke. This was investigated by examining the relationship between BBB alterations following transient ischemic stroke and expression of cellular adhesion molecule P-selectin using a combination of magnetic resonance molecular imaging (MRMI), intravital microscopy and immunohistochemistry. MRMI was performed using the contrast, gadolinium diethylenetriaminepentaacetic acid (Gd-DTPA) conjugated to Sialyl Lewis X (Sle ${ }^{x}$ where the latter is known to bind to activated endothelium via E- or P selectins. Middle cerebral artery occlusion was induced in male C57/BL 6 wildtype (WT) mice and P-selectin-knockout (KO) mice. At 24 hours following middle cerebral artery occlusion, $T_{1}$ maps were acquired prior to and following contrast injection. In addition to measuring P- and E-selectin expression in brain homogenates, alterations in BBB function were determined immunohistochemically by assessing the extravasation of immunoglobulin $\mathrm{G}(\mathrm{lgG})$ or staining for polymorphonuclear (PMN) leukocytes. In vivo assessment of BBB dysfunction was also investigated optically using intravital microscopy of the pial circulation following the injection of Fluorescein Isothiocyanate (FITC)-dextran (MW 2000 kDa).
\end{abstract}

Results: MRI confirmed similar infarct sizes and $T_{1}$ values at 24 hours following stroke for both WT and KO animals. However, the blood to brain transfer constant for Gd DTPA $\left(K_{g d}\right)$ demonstrated greater tissue extravasation of Gd DTPA in $W T$ animals than $K O$ mice $(P<0.03)$. In the $P$ selectin $K O$ mice, $\Delta T_{1}$ stroke $-\Delta T_{1}$ contralateral control cortex, decreased significantly in the Gd-DTPA(sLe ${ }^{x}$ group compared to Gd-DTPA, indicative of sLe ${ }^{X}$ mediated accumulation of the targeted contrast agent. Regarding BBB function, in the P-selectin KO mice compared to WT control mice, there was an attenuation in the extravasation of $\operatorname{lgG}(P<0.001)$, a trend for decreased FITC extravasation and less infiltration of PMN leukocytes $(P<0.001)$ thereby supporting the observed increase in $K_{g d}$ permeability in stroke brain of WT compared to KO mice.

Conclusion: P-selectin expression contributes to enhanced BBB dysfunction at 24 hours after transient focal cerebral ischemia.

\footnotetext{
* Correspondence: pabarber@ucalgary.ca

'Department of Clinical Neurosciences, University of Calgary, Calgary, Alberta, Canada
} 


\section{Background}

Leukocyte recruitment occurs after ischemia/reperfusion resulting in local tissue damage and compromised microvascular perfusion. Central to this process is the vascular endothelium expression of $\mathrm{P}$ - and E-selectin in the first few minutes to hours after cerebral ischemia, leading to polymorphonuclear (PMN) leukocyte or neutrophil migration into brain tissue, cytokine release and free radical-mediated damage [1]. During this time, blood brain barrier (BBB) injury can lead to an increase in vascular permeability and brain edema, exacerbating the initial ischemic injury [2]. Although neuroinflammatory processes contribute to BBB breakdown in many conditions [3], the link between early BBB dysfunction and endothelial cell activation in acute stroke is unclear. There is evidence that the inhibition of selectin adhesion molecules reduces brain injury and inhibits neutrophil and platelet accumulation after focal ischemia in mice $[4,5]$, but the mechanisms involved in this protection remain speculative [6].

We hypothesized that since there is evidence that Pselectin is a mediator of the early phase of leukocyte recruitment in acute ischemia it is also a major contributor to enhancing the early BBB dysfunction following stroke. Thus, we compared alterations in BBB permeability 24 hours following transient focal cerebral ischemia in wild type (WT) control mice to those in Pselectin knockout (KO) mice. The results demonstrate that following stroke P-selectin expression contributes to $\mathrm{BBB}$ injury as detected using a combination of magnetic resonance molecular imaging (MRMI), intravital microscopy and immunohistochemical methods. MRMI was performed using gadolinium (Gd) diethylenetriaminepentaacetic acid (DTPA) conjugated to a Sialyl Lewis $\mathrm{X}$ (Slex) - a mimetic of P-selectin glycoprotein ligand-1 tetrasaccharide sialyl Lewis $\mathrm{X}$ which mediates the binding of leukocytes and platelets to activated endothelium $[7,8]$.

\section{Methods}

\section{Animal Preparation}

All animal procedures were approved by the Animal Care Committees at the University of Calgary and the National Research Council of Canada. Transient middle cerebral artery occlusion of 30 minutes duration was induced in thirty four 30-35 gram male C57/BL 6 wildtype mice or $29 \mathrm{P}$-selectin-deficient or knockout mice (Jackson Laboratory) using the intraluminal suture occlusion method as described previously [9]. Control animals with sham surgery included all surgical steps except for middle cerebral artery occlusion $(\mathrm{n}=8)$. Temperature regulation during ischemia and reperfusion was achieved using an intra-abdominal telemetry probe
(Data Sciences International) with feedback to a heating pad to remove the potentially confounding influence of hypothermia during the reperfusion period. At 24 hours, the intra-abdominal probe was removed under aseptic conditions and temperature was then regulated by feedback from a rectal temperature probe during any subsequent procedures.

\section{Magnetic Resonance Imaging}

At 24 hours after stroke, animals were anesthetised with isoflurane for magnetic resonance (MR) imaging using a 9.4 T MR system equipped with a Bruker Avance console as described previously [9]. Briefly, $\mathrm{T}_{2}$ scans were acquired using a spin-echo sequence with a $2 \times 2 \mathrm{~cm}^{2}$ field of view, a $256 \times 128$ data matrix, a repetition time of $2750 \mathrm{~ms}$ and 24 echoes with $10 \mathrm{msec}$ echo-spacing for 8 slices $1 \mathrm{~mm}$ thick. $\mathrm{T}_{1}$ scans were acquired using a Contrast FAST sequence with a $2.5 \times 2.5 \mathrm{~cm}^{2}$ field of view, a $128 \times 128$ data matrix, a repetition time of 3.5 $\mathrm{ms}$, an echo time of $2 \mathrm{msec}$, a flip angle of $12^{\circ}$ and 20 inversion time points for one $1 \mathrm{~mm}$ thick slice through the infarct. $T_{1}$ and $T_{2}$ maps were determined using local imaging software (Marevisi, National Research Council).

For the experimental protocol, $\mathrm{T}_{1}$ and $\mathrm{T}_{2}$ scans were first acquired prior to contrast injection. Then, either DTPA (molecular weight $938 \mathrm{~g} / \mathrm{mol}$ ) or targeted contrast agent using a mimetic of Sialyl Lewis ${ }^{\mathrm{X}}$, coupled to Gd-DTPA (Gd-DTPA-(sLeX), molecular weight $1758 \mathrm{~g} /$ mol) was injected intravenously at a dose of $0.1 \mathrm{mmol}$ $\mathrm{Gd} / \mathrm{kg}$ body weight. Subsequently, $\mathrm{T}_{1}$ maps were acquired every 11 minutes for 55 minutes to allow GdDTPA-(sLeX) to bind to its target and to allow non-targeted (Gd-DTPA) contrast to at least partially clear from the circulation.

The first 33 minutes post injection was used to assess blood-brain barrier function by determining the transfer constant for Gd-DTPA into the brain $\left(\mathrm{K}_{\mathrm{Gd}}\right)$. $\mathrm{K}_{\mathrm{Gd}}$ was calculated using $T_{1}$ measures in the sagittal sinus and in regions of interest using a Patlak plot similar to that described by Ewing et al [10]. The regions of interest included the parietal cortex in the infarct core and the equivalent contralateral control cortex. $\mathrm{T}_{1}$ maps were also used to determine targeted contrast binding by calculating the quantity $\Delta \mathrm{T}_{1}$ Stroke $-\Delta \mathrm{T}_{1}$ Control Hemisphere which was defined as: [Post - pre-contrast mean $\mathrm{T}_{1}$ value of the stroke region] - [Post - pre-contrast mean $\mathrm{T}_{1}$ value of a corresponding region in the contralateral cortex]. After imaging, the animals were euthanized by intracardiac injection of sodium pentobarbital (70 mg/kg).

Infarct areas and volumes were calculated using the $\mathrm{T}_{2}$ MR images. The infarct area in each $\mathrm{T}_{2}$ slice was readily apparent as a region of increased $\mathrm{T}_{2}$. The calculation of the infarct area included a measure of the 
number of pixels in the ipsilateral hemisphere (with the exception of the ventricles) having a $T_{2}$ value greater than a threshold of 2 standard deviations above the mean $\mathrm{T}_{2}$ in the contralateral cortex. For each slice, the infarct area was corrected for edema (Ipsilateral infarct area (uncorrected)/((Ipsilateral hemisphere area/Contralateral hemisphere area)), and from this, the radius of this area was derived. Then the infarct and ipsilateral hemispheric volume between slices was calculated by the cone frustum volume approximation and the infarct and hemispheric tip volumes anteriorly and posteriorly were calculated by the cone volume approximation $[11,12]$.

\section{Protein and Immunohistochemical Tissue Analyses}

P- and E-selectin expression was measured in samples of cortical tissue homogenates from the hemispheres contralateral and ipsilateral to the infarct. These levels were quantified using commercially available enzyme-linked immunosorbent assay (ELISA) kits (Quantikine sP-selectin or sE-selectin ELISA Kit, R\&D Systems). Total protein in each sample was determined by the modified Lowry assay method.

After $24 \mathrm{~h}$, animals were injected with an overdose of sodium pentobarbital and perfused transcardially with $0.1 \mathrm{M}$ phosphate buffered saline followed by $4 \%$ paraformaldehyde. Brains were removed, dehydrated and embedded in paraffin blocks. Serial coronal paraffin sections $(10 \mu \mathrm{m})$ thick were cut and stained with hematoxylin and eosin for identification of infarcted tissue.

The presence of systemic immunoglobulin G (IgG) within brain was assessed immunohistochemically in 10 $\mu \mathrm{m}$ thick paraffin embedded coronal sections cut at the level of bregma $+1.2 \mathrm{~mm}$. Sections were stained with goat anti-mouse IgG antibody (1:200 concentration), using horseradish peroxidase-streptavidin (1:400 concentration) and diaminobenzidine for visualization. Sections from multiple treatment groups were processed together using standardized techniques for staining and measurement. Relative staining intensity was assessed using an Olympus BX 61 light microscope at 1.5× magnification. Intensity for IgG staining was quantified taking the grey levels of the darkest region in the ischemic hemisphere and subtracting it from the grey values measured in the corresponding region of darkest intensity in the contralateral hemisphere. With increasing BBB injury IgG extravasation increased resulting in darker staining and lower grey levels.

Immunohistochemical staining for polymorphonuclear (PMN) leukocytes was done on $30 \mu \mathrm{m}$ thick cryostat sections of brain. The sections were washed in PBS initially and between all incubations. First, sections were blocked for endogenous peroxidases by incubating with a mixture of $\mathrm{H}_{2} \mathrm{O}_{2}$ and methanol (3:27) for $15 \mathrm{~min}$.
Then the sections were blocked with $10 \%$ normal goat serum for $1 \mathrm{~h}$ at room temperature and incubated with a mouse monoclonal antibody to granulocytes (Abcam, Cambridge, MA, USA; 1:150) overnight at $4^{\circ} \mathrm{C}$. Subsequently, sections were incubated with anti-mouse biotinylated secondary antibody (Vectastain ABC Kit, Vector Labs, Burlingame, CA, USA) for $1.5 \mathrm{~h}$ at room temperature and incubated with Vectastain $A B C$ reagent (Vectastain ABC kit, Vector Labs, Burlingame, CA, USA) for $30 \mathrm{~min}$ at room temperature. The sections were incubated with 3, 3' diaminobenzidine tetrachloride-NickelCobalt (DAB-Ni-Co) substrate solution (Liquid DABBlack Substrate Kit, Vector Labs, Burlingame, CA, USA) until color developed, dehydrated and mounted. Using an Olympus BX 61 light microscope the labelled polymorphonuclear leucocytes were identified by their positive staining and morphologically by their characteristic bilobed nucleus. The sections were assessed at $\times 40 \mathrm{mag}$ nification in five specific predefined regions which included cingulate gyrus, parietal cortex, entorhinal cortex and two striatal regions (medial and lateral) [9].

\section{Intravital Microscopy}

Intravital microscopy was performed through a closed bone window in the anesthetized animal maintained at $36.5^{\circ} \mathrm{C}$ using a rectal temperature probe and heating pad. Images were acquired after tail-vein injection of FITC-dextran (MW 2000 kDa, 50 microlitres, 10 mg/ $\mathrm{mL}$ ). FITC extravasation from the pial vasculature was assessed every 5 minutes for 30 minutes with an exposure time of 20 seconds per image. Parameters for contrast, brightness and gamma correction remained unchanged throughout the measures.

\section{Statistical Analysis}

All data were analyzed by investigators blinded to the experimental group. Data is presented as mean \pm S.D. Comparisons between groups for stroke volume, mean infarct $\mathrm{T}_{1}$ value, $\mathrm{K}_{\mathrm{Gd}}$, IgG extravasation and PMN infiltration were assessed with a one-way ANOVA. MRMI contrast effects were assessed with a two-way ANOVA with repeated measures. Animals demonstrating FITC extravasation were documented and compared using the Freeman-Halton extension of a Fisher's exact test for 2 $\times 3$ tables. A p value of less than 0.05 was considered significant.

\section{Results}

\section{Stroke injury and Selectin expression}

All mice survived for 24 hours following stroke induced by transient middle cerebral artery occlusion. Cortical perfusion reductions during ischemia, measured using laser doppler flowmetry (LDF) were similar between groups with cortical perfusion decreasing to values of 
$14 \%, 17 \%, 19 \%$ and $16 \%$ of baseline for WT-Gd-Slex, WT-Gd, P-selectin KO-Gd-Slex and P selectin KO-Gd groups, respectively (n.s., $\mathrm{P}>0.7$, One-Way ANOVA). During reperfusion the LDF measurements were again similar between groups with values of $84 \%, 66 \%, 70 \%$ and $111 \%$ baseline flow upon reperfusion for WT-GdSlex, WT-Gd, P-selectin KO- Gd-Slex, and P selectin KO-Gd groups, respectively (n.s., $\mathrm{P}=0.3$, One-Way ANOVA).

All animals subjected to middle cerebral artery occlusion had ischemic infarction within the striatum and parietal cortex. Measurement of infarct size from MR images demonstrated that there was no substantial difference in the size of the stroke between the four experimental groups (figure $1 \mathrm{~A}$ ). $\mathrm{T}_{1}$ values, were increased in the ischemic region, however, mean values prior to contrast injection were similar between groups (Figure 1B).

The amount of P-selectin (ng/mg of protein) was determined in brain samples from brain ipsilateral and contralateral to the ischemia. Mean values were as follows: for wild type animals, $6.4 \pm 5.5$ in the ipsilateral cortex and $3.5 \pm 1.9$ in the contralateral cortex $(\mathrm{N}=6)$; for P-selectin knockouts, $0.19 \pm 0.3$ in the ipsilateral cortex and $0.06 \pm .08$ in the contralateral cortex $(\mathrm{N}=$ 2 ); and for shams, $0.5 \pm 0.2$ in the ipsilateral cortex and $0.64 \pm .09$ in the contralateral cortex $(\mathrm{N}=3)$. E-selectin expression was also measured in samples of brain ipsilateral and contralateral to the ischemia. Mean levels of E-selectin in $\mathrm{ng} / \mathrm{mg}$ of protein were: for wild type animals, $1.048 \pm 1.47$ in the ipsilateral cortex and $0.789 \pm$
0.73 in the contralateral cortex $(\mathrm{N}=6)$; for P-selectin knockout animals, $0.324 \pm 0.28$ in the ipsilateral cortex and $0.150 \pm 0.12$ in the contralateral cortex $(\mathrm{N}=6)$; and, for sham animals $0.0005 \pm .0001$ in ipsilateral cortex and $0.00054 \pm .00002$ mean contralateral cortex $(\mathrm{N}$ $=3$ ). Therefore the expression of P-selectin in ipsilateral cortex of wild type animals was significantly different from ipsilateral cortex of sham animals $(\mathrm{P}<0.001)$ with a trend for an ipsilateral-contralateral differences. Similarly the expression of E-selectin in ipsilateral cortex of $\mathrm{P}$ selectin KO animals was significantly increased compared to sham controls $(\mathrm{P}<0.03)$.

\section{Blood Brain Barrier Function and Targeted Contrast Effects}

Permeability of the BBB was first assessed using the transfer constant for Gd-DTPA $\left(\mathrm{K}_{\mathrm{gd}}\right)$ into brain of a group of wild-type mice compared to a group of $\mathrm{P}$ selectin deficient mice (Figure 2). Mean $\mathrm{K}_{\mathrm{Gd}}$ values were greater in the ischemic cortex in wild-type mice compared to P-selectin knockouts, demonstrating enhanced BBB dysfunction in the former group.

Selectivity of contrast accumulation or binding was also assessed by MRMI using Gd-DTPA-SleX and monitoring $\mathrm{T}_{1}$ changes over 55 minutes in these animals. In knockout mice, there was a significant decrease in $\left(\Delta \mathrm{T}_{1}\right.$ Stroke- $\Delta \mathrm{T}_{1}$ Control Hemiphere $)$ in the Gd-DTPA- $\left(\mathrm{sLe}^{\mathrm{X}}\right)$ group $(\mathrm{n}=5)$ compared to $\mathrm{Gd}$ DTPA $(\mathrm{n}=5)$ at 33,44 and 55 minutes after injection (Figure 3). No such difference could be identified in wild-type animals administered either Gd-DTPA or
A

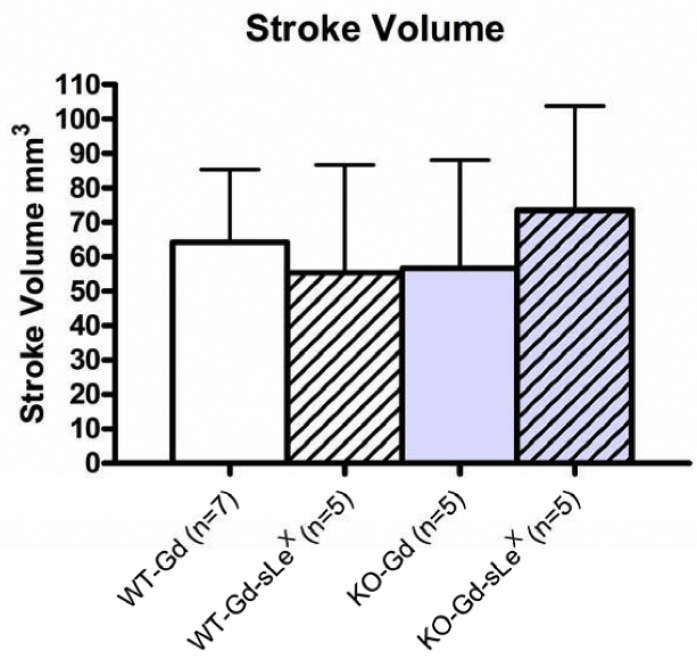

B

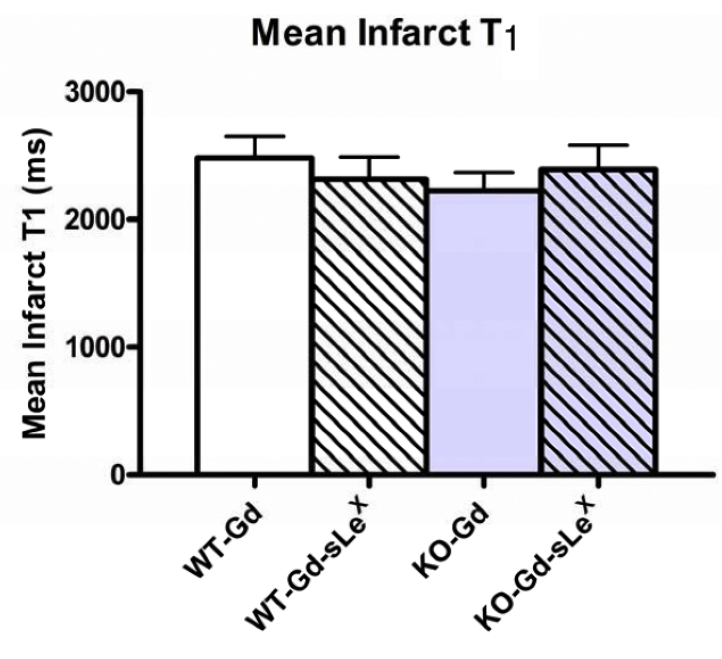

Figure 1 Effects of transient cerebral ischemia on mean infarct volume and mean cerebral. $T_{1}$. Twenty four hours following 30 min. of middle cerebral artery occlusion infarct volumes (mean \pm SD) were similar in wild-type mice (WT) or P-selectin knockout (KO) mice administered either gadolinium-DTPA (Gd) or Gd-DTPA conjugated with sialyl Lewis X (sLex) (A). Also, shown are $T_{1}$ values in the ischemic brain prior to contrast administration. (mean \pm SD) (B). Values were similar in all groups (n.s.). 


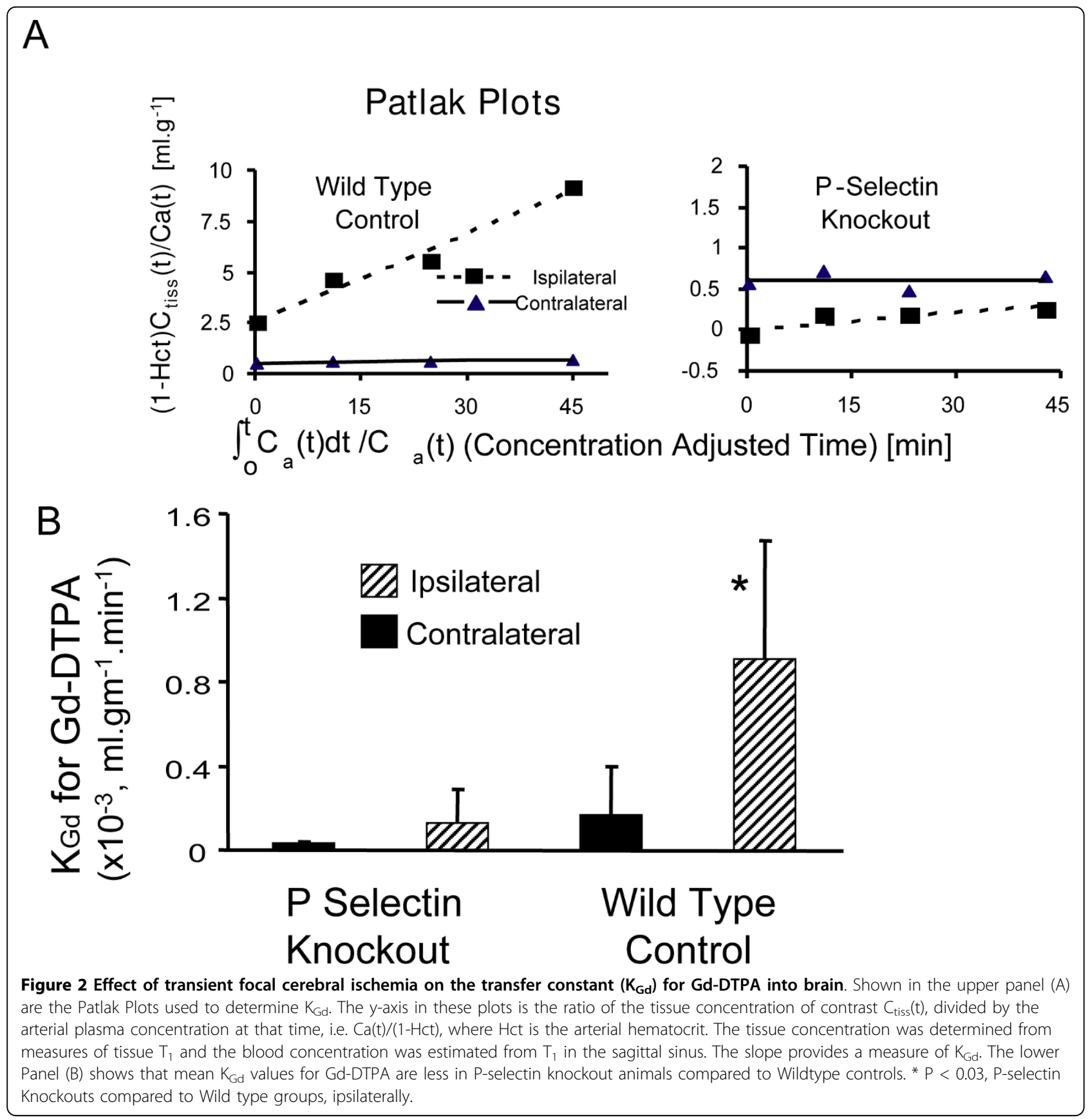

Gd-DTPA- $\left.\left(\mathrm{sLe}^{\mathrm{X}}\right)\right)$. Because the Sle ${ }^{\mathrm{X}}$ conjugated contrast agent binds to both $\mathrm{E}$ and $\mathrm{P}$-selectin and P-selectin is essentially not expressed in the brain of $\mathrm{KO}$ mice, these results suggest there is E-selectin-mediated binding to endothelium of the conjugated contrast agent following stroke.

\section{Immunoglulin G or Dextran extravasation and} Polymorphonuclear Leukocyte Infiltration

IgG extravasation was evaluated in 4 sham surgery mice, 10 wild-type mice and 6 knockout mice (Figure 4A, B).
The mean contralateral - ipsilateral differences in optical density were significantly higher in the wild-type group than in either the sham surgery or knockout groups, indicative of a more severe BBB injury in the wild-type animals $(\mathrm{p}<0.0001)$.

The infiltration of polymorphonuclear (PMN) leukocytes into the brain indicated differences between groups. In the WT mice $(\mathrm{N}=7)$, the number of PMNs in the ipsilateral hemisphere was significantly higher than in those of the $\mathrm{KO}(\mathrm{N}=6), \mathrm{p}<0.001)$ and sham mice $(\mathrm{N}=4, \mathrm{p}<0.001)$ (Figure $4 \mathrm{C})$. 


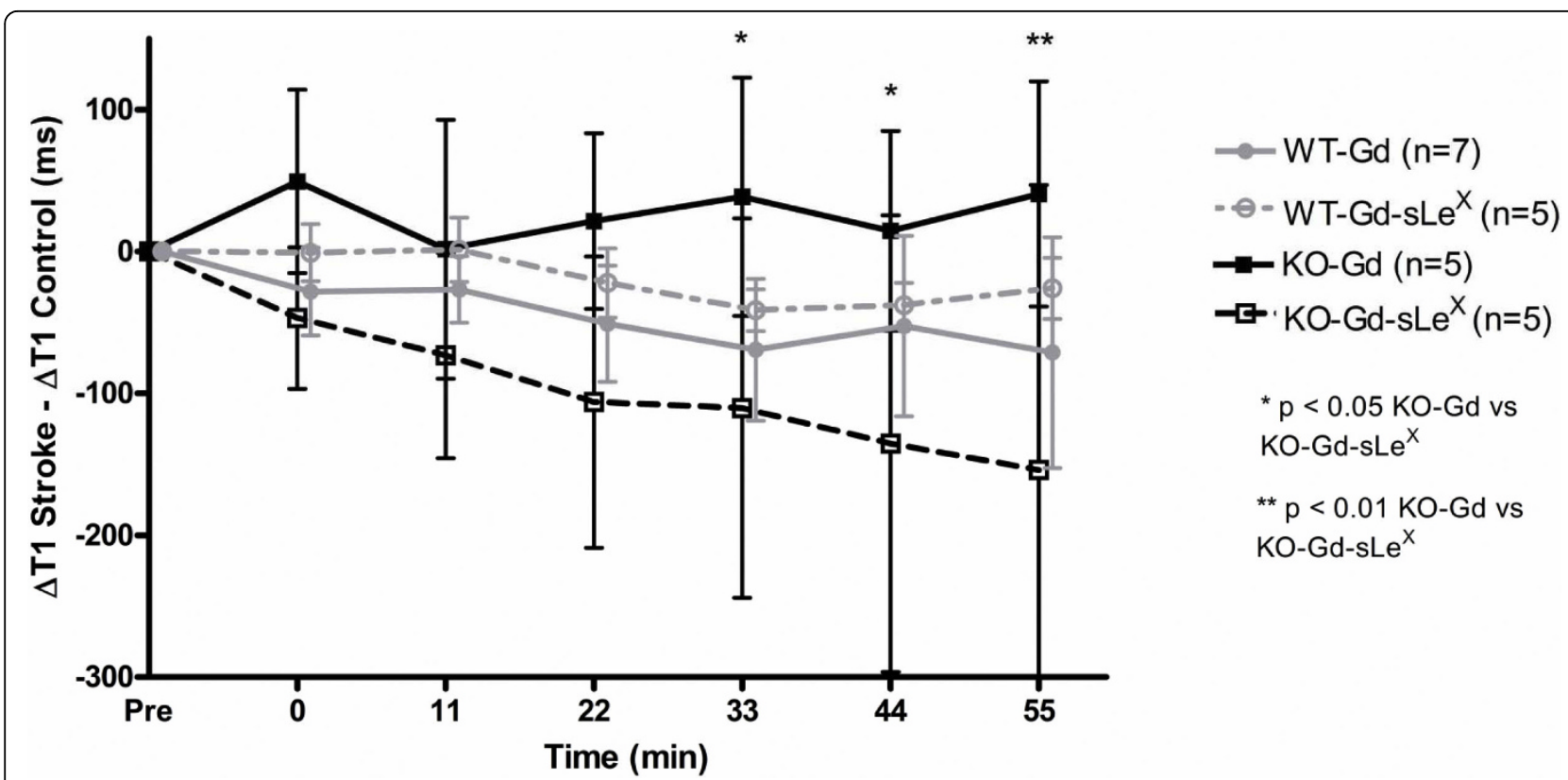

Figure 3 Magnetic resonance molecular imaging with Gd-DTPA-(sLe ${ }^{\mathbf{x}}$ ). In knockout (KO) mice there was increasing effects of targeted contrast Gd-DTPA-(sLe $\left.{ }^{x}\right)$ on tissue $T_{1}$ levels in the stroke compared to the contralateral control hemisphere not observed with non-targetted contrast or Gd-DTPA. ${ }^{*} P<0.05$ and ${ }^{* *} P<0.01$. In wild type (WT) animals the administration of Gd-DTPA-(sLe $\left.{ }^{x}\right)$ or Gd-DTPA resulted in a decrease in $T_{1}$ in the stroke compared to contralateral control hemisphere in both groups but there no statistical difference between groups.

Intravital microscopy was performed in 4 sham surgery mice, 9 wild-type and 5 knockout mice at 24 hours after reperfusion (Figure 4D). Fluorescein isothiocyanate (FITC)-dextran was injected and its extraluminal concentration monitored. Increased FITC fluorescence, indicative of enhanced vascular permeability, was observed by 30 minutes after FITC injection in 8/9 WT mice $(89 \%)$ and in $2 / 5 \mathrm{KO}$ mice $(40 \%)$ but in none of the sham mice $(0 / 4)$ (Fisher Exact $3 \times 2$ table, $\mathrm{p}=$ 0.002). The frequency of FITC extravasation for WT mice was greater than WT sham animals (Fishers Exact $\mathrm{P}<0.01)$, but there was no difference in frequency between $\mathrm{KO}$ mice and WT sham (NS, P > 0.5). There was a strong trend for more frequent FITC extravasation observed in WT mice compared to $\mathrm{KO}$ mice (Fischer's Exact two tailed test $\mathrm{P}<0.095$ ).

\section{Discussion}

Blood brain barrier impairment is a well-known marker of secondary cerebral injury following acute stroke associated with increases in a propensity for hemorrhagic transformation in infarcted tissue. The novel and important finding in the present study is that P-selectin expression contributes to BBB dysfunction at 24 hours after acute stroke, thus illustrating an expanded role of endothelial dysfunction as a key process in the early development of ischemic injury. In our study, early endothelial activation was evident by detecting significant cortical selectin expression whereas under normal physiologic conditions neither cerebral P- or Eselectin are detected in substantial amounts [13]. The extravasation of Gd-DTPA in the wild-type but not knockout mouse indicates that P-selectin expression is associated with more severe $\mathrm{BBB}$ impairment, despite similar infarct size between the two groups. Additionally, the similar $\mathrm{T}_{1}$ values in wild-type and knockout mice suggest comparable infarct water content and similar parenchymal injury severity [14]. Therefore, despite similar infarct extent and indicators of parenchymal injury, the E and/or P-selectin binding of Gd-DTPA$\left(\mathrm{sLe}^{\mathrm{X}}\right)$ in the knockout mouse was confounded less by BBB breakdown than in the wild-type mouse, and the lack of substantial P-selectin expression in these animals supports a possible E-selectin mediated target for the Sialyl Lewis X conjugated contrast agent.

Although P-selectin expression on endothelial cell and platelet membranes has been reported to be pivotal for leukocyte recruitment during the early stroke period [1], the role of selectin adhesion molecules in promoting brain edema and BBB injury is unclear. Ruehl et al suggested that P-selectin blockade with fucoidin resulted in less brain edema at 24 hours after 4 hour middle cerebral artery occlusion [6]. In our study, there was evidence that $\mathrm{BBB}$ injury was more severe in the wild-type mouse as indicated by greater extravasation of GdDTPA and a somewhat higher frequency for 


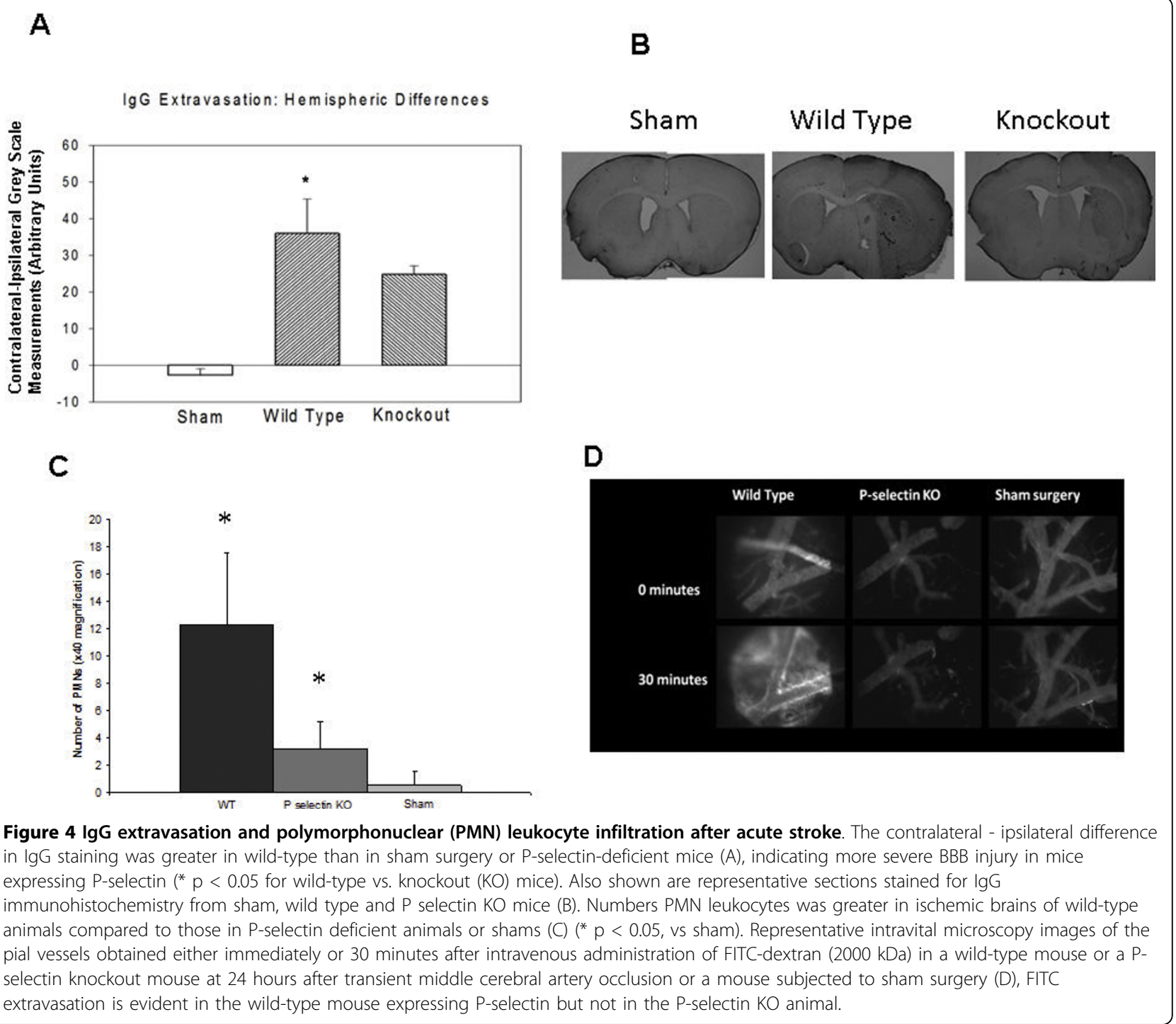

extravasation of FITC than in $\mathrm{KO}$ mice raising the possibility of differential contributions of selectins to BBB damage. We also show that PMN infiltration into the infarct region is greater in the wild-type animals than the P-selectin knockout animals, implicating P-selectin as a major contributor to PMN recruitment and ultimately infiltration into the stroke region. Our preliminary data would also support the involvement of both Pselectin and PMN migration in early BBB breakdown. Further work will be required to elucidate the precise molecular mechanisms involved.

Previous studies have demonstrated that while ICAM1 and E-selectin expression were necessary for neutrophil transmigration across a human brain microvessel endothelial cell layer, antibody blockade of ICAM-1 did not reduce vessel permeability to horseradish peroxidase despite dramatically decreased neutrophil migration
[15]. This suggests different mechanisms involving ICAM-1 and possibly other cellular adhesion molecules underlie neutrophil migration and BBB injury. This dissociation between neutrophil-mediated cerebral injury and BBB dysfunction has also been demonstrated in traumatic brain injury[16]. While neutrophil accumulation and E-selectin expression appear to mediate blood vessel permeability to some extent after trauma, similar to our current results, mice deficient in both P-selectin and ICAM-1 had less brain edema than wild-type mice, suggesting that trauma-induced BBB injury is influenced preferentially by specific neuroinflammatory markers. Following cerebral ischemia/reperfusion, TNF- $\alpha$ levels are increased within two hours [17], followed by a peak in P-selectin expression at 6 hours and a later peak in E-selectin expression between 6 and 12 hours [18]. BBB breakdown after middle cerebral artery occlusion and 
reperfusion becomes evident at 6 hours and appears to reach its maximal extent by 24 hours [19]. The poststroke progressive sequence of TNF- $\alpha$ expression, selectin upregulation and BBB injury suggests a chronological link between these events. Although many stroke patients present to emergency care at a time that is beyond the therapeutic window for thrombolysis, there remains the possibility of attenuating secondary cerebral injury in these patients by protecting the BBB. The present findings suggest a role for P-selectin as an early mediator of $\mathrm{BBB}$ injury and suggest an additional rationale to develop anti-inflammatory P-selectin targeted therapy to mitigate stroke-induced vascular injury.

The evidence that $\mathrm{P}$ selectin may be involved in increasing the size of the stroke lesion remains poorly substantiated. In our study, although we were able to demonstrate an effect of reduced BBB breakdown in P selectin knockout animals, we were not able to show an effect of reducing stroke volume. The literature that specifically addresses the issue of whether attenuating Pselectin expression reduces infarct size is sparse. Connolly et al [20] reported that isolated, purified, radiolabelled neutrophils accumulated in post-ischemic brain and that there were fewer neutrophils accumulating in P-selectin-deficient mice. In a model of cerebral ischemia reperfusion, P-selectin knockout mice exhibited a reduction in infarct volume, better functional outcome and a better return of cerebral blood flow after ischemia [20]. Similarly, blocking antibodies for P-selectin reduced infarct size and hemorrhagic transition in ischemia reperfusion [21]. The results of these studies are at least in part consistent with those observed presently regarding the beneficial effects of reduced P-selectin expression on BBB function. In moving towards clinical translation, the role for P-selectin in ischemic injury induced blood brain barrier dysfunction would need confirmation in additional animal studies that demonstrate the effectiveness on long-term and behavioural outcomes of antagonising P-selectin expression for example using either P-selectin antibody or P-selectin peptide.

\section{Conclusions}

The results indicate that elevated P-selectin expression following ischemia-reperfusion contributes to enhanced BBB dysfunction at 24 hours after transient focal cerebral ischemia despite a lack of appreciable effect on overall infarct volume.

\section{Acknowledgements}

The authors thank T. Foniok and D. Kirk for their expert assistance in the acquisition of the MR images. This study was supported by a grant from the Heart and Stroke Foundation of Alberta. AYJ was supported by a Focus on Stroke CIHR/RxD Collaborative Research Program Fellowship and by an
Alberta Heritage Foundation for Medical Research Clinical Fellowship Award. P.A.B. was supported by the Alberta Heritage Foundation for Medical Research and the Heart and Stroke Foundation of Canada.

\section{Author details}

'Department of Clinical Neurosciences, University of Calgary, Calgary, Alberta, Canada. ${ }^{2}$ National Research Council of Canada, Institute for Biodiagnostics (West), Calgary, Alberta, Canada. ${ }^{3}$ Department of General, Organic and Biomedical Chemistry, NMR and Molecular Imaging Laboratory, Université de Mons-Hainaut, Mons, Belgium.

\section{Authors' contributions}

AJ carried out the animal model, organised the data acquisition and analysis and wrote the manuscript. UT was involved in the conceptualization of the study, data analysis and acquisition, in addition to contributing to critical appraisal and writing of the manuscript. DR performed the in vivo stroke animal studies and contributed to data acquisition and analysis. JK was involved in data acquisition and tissue processing as well as statistical analysis. RM designed MR molecular contrast agent and provided critical appraisal of the manuscript. JLP was involved in the in vivo stroke studies and tissue processing as well as data analysis. SB was intimately involved in the MRI contrast agent development and the critical appraisal of the manuscript. PB conceptualized the study design, was involved in data analysis, coordinated all aspects of the study and was involved in coauthoring and critically appraising the manuscript. All authors read and approved the final manuscript.

\section{Competing interests}

The authors declare that they have no competing interests.

Received: 9 June 2009

Accepted: 2 February 2010 Published: 2 February 2010

\section{References}

1. Huang J, Upadhyay UM, Tamargo RJ: Inflammation in stroke and focal cerebral ischemia. Surg Neurol 2006, 66:232-245.

2. Latour LL, Kang DW, Ezzeddine MA, Chalela JA, Warach S: Early blood-brain barrier disruption in human focal brain ischemia. Ann Neurol 2004, 56:468-477.

3. Persidsky Y, Ramirez SH, Haorah J, Kanmogne GD: Blood-brain barrier: structural components and function under physiologic and pathologic conditions. J Neuroimmune Pharmacol 2006, 1:223-236.

4. Ishikawa M, Cooper D, Arumugam TV, Zhang JH, Nanda A, Granger DN: Platelet-leukocyte-endothelial cell interactions after middle cerebral artery occlusion and reperfusion. J Cereb Blood Flow Metab 2004, 24:907-915.

5. Ishikawa M, Cooper D, Russell J, Salter JW, Zhang JH, Nanda A, Granger DN: Molecular determinants of the prothrombogenic and inflammatory phenotype assumed by the postischemic cerebral microcirculation. Stroke 2003, 34:1777-1782.

6. Ruehl ML, Orozco JA, Stoker MB, McDonagh PF, Coull BM, Ritter LS: Protective effects of inhibiting both blood and vascular selectins after stroke and reperfusion. Neurol Res 2002, 24:226-232.

7. Kogan TP, Dupre B, Keller KM, Scott IL, Bui H, Market RV, Beck PJ, Voytus JA, Revelle BM, Scott D: Rational design and synthesis of small molecule, non-oligosaccharide selectin inhibitors: (alpha-D-mannopyranosyloxy) biphenyl-substituted carboxylic acids. J Med Chem 1995, 38:4976-4984.

8. Boutry S, Burtea C, Laurent S, Toubeau G, Vander EL, Muller RN: Magnetic resonance imaging of inflammation with a specific selectin-targeted contrast agent. Magn Reson Med 2005, 53:800-807.

9. Barber PA, Foniok T, Kirk D, Buchan AM, Laurent S, Boutry S, Muller RN, Hoyte L, Tomanek B, Tuor UI: MR molecular imaging of early endothelial activation in focal ischemia. Ann Neurol 2004, 56:116-120.

10. Ewing JR, Knight RA, Nagaraja TN, Yee JS, Nagesh V, Whitton PA, Li L, Fenstermacher JD: Patlak plots of Gd-DTPA MRI data yield blood-brain transfer constants concordant with those of $14 \mathrm{C}$-sucrose in areas of blood-brain opening. Magn Reson Med 2003, 50:283-292.

11. Perez-Trepichio AD, Furlan AJ, Little JR, Jones SC: Hydroxyethyl starch 200/ 0.5 reduces infarct volume after embolic stroke in rats. Stroke 1992, 23:1782-1791. 
12. Kharlamov A, Zivkovic I, Polo A, Armstrong DM, Costa E, Guidotti A: LIGA20, a lyso derivative of ganglioside GM1, given orally after cortical thrombosis reduces infarct size and associated cognition deficit. Proc Natl Acad Sci USA 1994, 91:6303-6307.

13. Eppihimer MJ, Russell J, Anderson DC, Wolitzky BA, Granger DN: Endothelial cell adhesion molecule expression in gene-targeted mice. Am J Physiol 1997, 273:H1903-H1908.

14. Barbier EL, Liu L, Grillon E, Payen JF, Lebas JF, Segebarth C, Rémy C: Focal brain ischemia in rat: acute changes in brain tissue $\mathrm{T} 1$ reflect acute increase in brain tissue water content. NMR Biomed 2005, 18:499-506.

15. Wong D, Prameya R, Dorovini-Zis K: Adhesion and migration of polymorphonuclear leukocytes across human brain microvessel endothelial cells are differentially regulated by endothelial cell adhesion molecules and modulate monolayer permeability. I Neuroimmunol 2007, 184:136-148.

16. Whalen MJ, Carlos TM, Dixon CE, Robichaud P, Clark RS, Marion DW, Kochanek PM: Reduced brain edema after traumatic brain injury in mice deficient in P-selectin and intercellular adhesion molecule-1. J Leukoc Biol 2000, 67:160-168.

17. Pan W, Kastin AJ: Tumor necrosis factor and stroke: role of the bloodbrain barrier. Prog Neurobiol 2007, 83:363-374

18. Danton GH, Dietrich WD: Inflammatory mechanisms after ischemia and stroke. J Neuropathol Exp Neurol 2003, 62:127-136.

19. Yang GY, Gong C, Qin Z, Liu XH, Lorris BA: Tumor necrosis factor alpha expression produces increased blood-brain barrier permeability following temporary focal cerebral ischemia in mice. Brain Res Mol Brain Res 1999, 69:135-143.

20. Connolly ES Jr, Winfree CJ, Prestigiacomo CJ, Kim SC, Choudhri TF, Hoh BL, Naka Y, Solomon RA, Pinsky DJ: Exacerbation of cerebral injury in mice that express the P-selectin gene: identification of P-selectin blockade as a new target for the treatment of stroke. Circ Res 1997, 81:304-310.

21. Goussev AV, Zhang Z, Anderson DC, Chopp M: P-selectin antibody reduces hemorrhage and infarct volume resulting from MCA occlusion in the rat. J Neurol Sci 1998, 161:16-22.

doi:10.1186/1471-2202-11-12

Cite this article as: Jin et al:: Reduced blood brain barrier breakdown in P-selectin deficient mice following transient ischemic stroke: a future therapeutic target for treatment of stroke. BMC Neuroscience 2010 11:12

\section{Submit your next manuscript to BioMed Central and take full advantage of:}

- Convenient online submission

- Thorough peer review

- No space constraints or color figure charges

- Immediate publication on acceptance

- Inclusion in PubMed, CAS, Scopus and Google Scholar

- Research which is freely available for redistribution 